\title{
Membrane paradigm and holographic DC conductivity for nonlinear electrodynamics
}

\author{
Xiaobo Guo, ${ }^{1, *}$ Peng Wang, ${ }^{2, \dagger}$ and Haitang Yang ${ }^{2, \$}$ \\ ${ }^{1}$ School of Science, Southwest University of Science and Technology, \\ Mianyang, 621010, People's Republic of China \\ ${ }^{2}$ Center for Theoretical Physics, College of Physical Science and Technology, Sichuan University, \\ Chengdu, 610064, People's Republic of China
}

(Received 8 January 2018; published 16 July 2018)

\begin{abstract}
The membrane paradigm is a powerful tool to study the properties of black hole horizons. We first explore the properties of the nonlinear electromagnetic membrane of black holes. For a general nonlinear electrodynamics field, we show that the conductivities of the horizon usually have off-diagonal components and depend on the normal electric and magnetic fields on the horizon. Via the holographic duality, we find a model-independent expression for the holographic DC conductivities of the conserved current dual to a probe nonlinear electrodynamics field in a neutral and static black brane background. It shows that these DC conductivities only depend on the geometric and electromagnetic quantities evaluated at the horizon. We can also express the DC conductivities in terms of the temperature, charge density, and magnetic field in the boundary theory, as well as the values of the couplings in the nonlinear electrodynamics at the horizon.
\end{abstract}

DOI: 10.1103/PhysRevD.98.026021

\section{INTRODUCTION}

Black holes are among the most intriguing concepts of general relativity. The event horizon of a black hole is a puzzling and fascinating object, in that natural descriptions of physics often have trouble accommodating the horizon. One of the challenges of extending theories to the horizon is defining boundary conditions on the horizon. The horizon is a null hypersurface, which possesses a singular Jacobian and both a normal and tangential vector field. If one believes that (1) the effective number of degrees of freedom within a few Planck lengths away from the horizon is very small and (2) the interior of the black hole is a classically inaccessible region to an outside observer, an effective timelike membrane can be put just outside the horizon to have boundary conditions defined on it, instead of the horizon. These observations form the basis of the membrane paradigm for black holes.

The membrane paradigm was proposed and developed by Thorne and his Caltech colleagues in a series of papers [1-5]. Later, a more systematic action-based derivation was

\footnotetext{
*guoxiaobo@swust.edu.cn

pengw@scu.edu.cn

*hyanga@scu.edu.cn
}

Published by the American Physical Society under the terms of the Creative Commons Attribution 4.0 International license. Further distribution of this work must maintain attribution to the author(s) and the published article's title, journal citation, and DOI. Funded by SCOAP ${ }^{3}$. obtained by Parikh and Wilczek in [6], which could determine membrane properties for various field theories. The membrane paradigm was originally developed to serve as an efficient computational tool useful in dealing with some astrophysical physics in black hole backgrounds [7-9]. On the other hand, the membrane paradigm is also useful to study microscopic properties of black hole horizons. For example, the membrane paradigm predicts that black hole horizons are the fastest scramblers in nature $[10,11]$. In particular, the authors of [12] studied the electromagnetic membrane properties of the horizon and considered a charged particle dropping onto the horizon in the framework of Maxwell-Chern-Simons theory. They found that the black hole horizon behaved as a Hall conductor, and there were vortices introduced to the way perturbations scramble on the horizon.

The AdS/CFT duality $[13,14]$ conjectures a connection between a strongly coupled gauge theory in $d$ dimensions on the boundary and a dual weakly coupled gravity in $(d+1)$-dimensional bulk spacetime. Recently, a renewed interest has emerged in the study of the membrane paradigm in the context of the AdS/CFT duality. It has been shown that the membrane paradigm fluid on the black hole horizon is conjectured to give the low-frequency limit of linear response of a strongly coupled quantum field theory at a finite temperature [15-18]. In particular, by identifying the currents in the boundary theory with radially independent quantities in the bulk, the authors of [17] showed that the low-frequency limit of the boundary theory transport coefficients could be expressed in terms of 
geometric quantities evaluated at the horizon. This method was later extended to calculate the DC conductivity in the presence of momentum dissipation [19-22], where the zero mode of the current, not the current itself, did not evolve in the radial direction. Specifically, the DC thermoelectric conductivity has recently been obtained by solving a system of Stokes equations on the black hole horizon for a charged fluid in Einstein-Maxwell theory [23]. Later, the technology of [23] was extended to various theories, e.g., Einstein-Maxwell-scalar theory [24] and including a $\theta F \wedge F$ term in the Lagrangian [25].

Nonlinear electrodynamics (NLED) are effective models incorporating quantum corrections to Maxwell electromagnetic theory. Among the various NLED, there are two wellknown ones. The first is the Heisenberg-Euler effective Lagrangian that contains logarithmic terms [26]. These terms describe the vacuum polarization effects and take into account one-loop quantum corrections to QED. The second is Born-Infeld electrodynamics that incorporates maximal electric fields and smooths divergences of the electrostatic self-energy of point charges [27]. Coupling NLED to gravity, various NLED charged black holes were derived in a number of papers [28-32]. Some of these black holes are nonsingular exact black hole solutions [29]. In the framework of AdS/CFT duality, the shear viscosity was calculated in Einstein-Born-Infeld gravity [33]. Holographic superconductors were studied in several NLED-gravity theories [34-37]. In [38], the holographic conductivity for the black brane solutions in the massive gravity with a power-law Maxwell field was numerically investigated. A class of holographic models for Mott insulators, whose gravity dual contains NLED, was studied in [39]. The authors of [40,41] used the AdS/CFT correspondence to compute the DC conductivities holographically from a probe Born-Infeld action. Recently, the properties of magnetotransport in holographic DiracBorn-Infeld models were discussed in a probe case [42], taking into account the effects of backreaction on the geometry [43].

In this paper, we will consider a neutral and static black brane background with a probe NLED field and its dual theory. The aim of the paper is to study the NLED electromagnetic membrane properties and find a modelindependent expression for the holographic DC conductivities of the dual conserved current in the boundary theory. Specifically, we give a quick review of nonlinear electromagnetic fields in the curved spacetime in Sec. II. In Sec. III, we use the membrane paradigm to compute the conductivities of the stretched horizon. Unlike Maxwell or Maxwell-Chern-Simons theories, we find that, for a general NLED field, the conductivities would usually depend on the normal electric and magnetic fields on the stretched horizon. In Sec. IV, we consider a charged point particle infalling into the horizon in Rindler space. It shows that effects of NLED do not affect the charge density on the stretched horizon or the scrambling time, but only change the way the charge scrambles. In Sec. V, the DC conductivities of the dual conserved current are calculated in the framework of gauge/gravity duality. We show that these DC conductivities usually depend on both the geometry and values of the couplings in NLED at the black hole horizon as well as the probe charge density and magnetic field in the boundary theory. In Sec. VI, we conclude with a brief discussion of our results. We use the convention that the Minkowski metric has the signature of the metric $(-+++)$ in this paper.

\section{NONLINEAR ELECTRODYNAMICS}

In this section, we will briefly review nonlinear electromagnetic fields in the curved spacetime, mainly in order to define terms and notation and derive formulas for later use. Let us consider the action of a nonlinear electromagnetic field $A_{a}$ in a $(3+1)$-dimensional manifold $\mathcal{M}$,

$$
S=\int_{\mathcal{M}} d^{4} x \sqrt{-g}\left[\mathcal{L}(s, p)+J^{a} A_{a}\right]
$$

where we build two independent nontrivial scalars using $F_{a b}$ and none of its derivatives:

$$
\begin{aligned}
& s=-\frac{1}{4} F^{a b} F_{a b}, \\
& p=-\frac{1}{8} \epsilon^{a b c d} F_{a b} F_{c d} ;
\end{aligned}
$$

the field strength is defined by $F_{a b}=\partial_{a} A_{b}-\partial_{b} A_{a} ; \epsilon^{a b c d} \equiv$ $-\left[\begin{array}{llll}a & b & c & d\end{array}\right] / \sqrt{-g}$ is a totally antisymmetric Lorentz tensor,

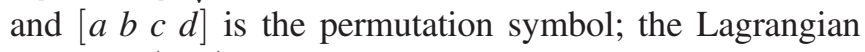
density $\mathcal{L}(s, p)$ is an arbitrary function of $s$ and $p$; and $J^{a}$ is the external current. We also assume that the NLED Lagrangian would reduce to the form of a MaxwellChern-Simons Lagrangian for small fields:

$$
\mathcal{L}(s, p) \approx g(x) s+\theta(x) p,
$$

where, for later convenience, we define

$$
g(x) \equiv \mathcal{L}^{(1,0)}(0,0) \quad \text { and } \quad \theta(x) \equiv \mathcal{L}^{(0,1)}(0,0) .
$$

Here we allow coordinate-dependent couplings in $\mathcal{L}(s, p)$. The equations of motion obtained from the action (1) are

$$
\nabla_{a} G^{a b}+J^{b}=0
$$

where we define

$G^{a b}=-\frac{\partial \mathcal{L}(s, p)}{\partial F_{a b}}=\frac{\partial \mathcal{L}(s, p)}{\partial s} F^{a b}+\frac{1}{2} \frac{\partial \mathcal{L}(s, p)}{\partial p} \epsilon^{a b c d} F_{c d}$. 
Meanwhile, from the definition of $F_{a b}$, the field strength also satisfies the Bianchi identity

$$
F_{[a b, c]}=\frac{1}{3}\left(F_{a b, c}+F_{b c, a}+F_{c a, b}\right)=0 .
$$

The electric and magnetic fields measured by an observer with four-velocity $U^{a}$ are given by

$$
\begin{aligned}
E^{a} & =F^{a b} U_{b}, \\
B^{a} & =\frac{1}{2} \epsilon^{b a c d} F_{c d} U_{b},
\end{aligned}
$$

Note that the fields $E^{a}$ and $B^{a}$ are three-vectors since $E^{a} U_{a}=B^{a} U_{a}=0$. The variables $s$ and $p$ can be rewritten in terms of $E^{a}$ and $B^{a}$ :

$$
\begin{aligned}
& s=\frac{1}{2}\left(E^{a} E_{a}-B^{a} B_{a}\right), \\
& p=-E^{a} B_{a} .
\end{aligned}
$$

Similarly for $G^{a b}$, we can define auxiliary fields $D^{a}$ and $H^{a}$ :

$$
\begin{aligned}
D^{a} & =G^{a b} U_{b}, \\
H^{a} & =\frac{1}{2} \epsilon^{b a c d} G_{c d} U_{b},
\end{aligned}
$$

which are related to $E^{a}$ and $B^{a}$ by

$$
\begin{aligned}
D^{a} & =\frac{\partial \mathcal{L}(s, p)}{\partial s} E^{a}-\frac{\partial \mathcal{L}(s, p)}{\partial p} B^{a}, \\
H^{a} & =\frac{\partial \mathcal{L}(s, p)}{\partial s} B^{a}+\frac{\partial \mathcal{L}(s, p)}{\partial p} E^{a} .
\end{aligned}
$$

The electromagnetic four-current $J^{a}$ can be split into the charge density $\rho$ and current density $\mathbf{J}^{a}$ measured by the observer:

$$
\begin{gathered}
\rho=-J^{a} U_{a}, \\
\mathbf{J}^{a}=J^{a}-\sigma U^{a},
\end{gathered}
$$

where $\mathbf{J}^{a}$ is a two-vector since $\mathbf{J}^{a} U_{a}=\mathbf{J}^{a} n_{a}=0$.

Born-Infeld electrodynamics is described by the Lagrangian density

$$
\mathcal{L}_{\mathrm{BI}}(s, p)=-b^{2} \sqrt{1-\frac{2 s}{b^{2}}-\frac{p^{2}}{b^{4}}}+b^{2},
$$

where the coupling parameter $b$ is related to the string tension $\alpha^{\prime}$ as $b=1 / 2 \pi \alpha^{\prime}$. For small fields $s, p \ll b^{2}$, we can recover the Maxwell Lagrangian. A simple example of an electrodynamics Lagrangian with a logarithmic term has the form

$$
\mathcal{L}_{\mathrm{Log}}(s, p)=-b^{2} \log \left(1-\frac{s}{b^{2}}\right)
$$

This Lagrangian also reduces to the Maxwell case in the limit $b \rightarrow \infty$.

As a simple example, let us calculate the electric and magnetic fields of a point charge in four-dimensional Minkowski space. In Minkowski space, Eqs. (5) and (7) become

$$
\begin{aligned}
\nabla \times \vec{E} & =-\frac{\partial \vec{B}}{\partial t}, \\
\nabla \cdot \vec{B} & =0, \\
\nabla \cdot \vec{D} & =\rho, \\
\nabla \times \vec{H} & =\vec{j}+\frac{\partial \vec{D}}{\partial t},
\end{aligned}
$$

where $\rho=Q \delta^{3}(\vec{r})$ and $\vec{j}=0$ for a point charge sitting at $\vec{r}=0$. Since $\partial \vec{E} / \partial t=\partial \vec{B} / \partial t=0$ in this case, Eqs. (15) lead to

$$
\vec{D}=\frac{Q}{4 \pi r^{2}} \hat{r} \quad \text { and } \quad B=0 .
$$

Considering $s=E^{2} / 2$ and $p=0$, we can solve Eqs. (11) for $\vec{E}$ and find that

$$
\vec{E}=\frac{Q}{4 \pi r^{2}} F\left(\frac{Q}{4 \pi r^{2}}\right) \hat{r}
$$

where $y(x)=x F(x)$ is the inverse of the function $x(y)=\mathcal{L}^{(1,0)}\left(\frac{y^{2}}{2}, 0\right) y$. For example, one has

$F(x)=\left\{\begin{array}{ll}\frac{1}{\sqrt{1+x^{2} / b^{2}}} \quad \text { Born-Infeld electrodynamics } \\ \frac{-1+\sqrt{1+2 x^{2} / b^{2}}}{x^{2} / b^{2}} & \text { Logarithmic electrodynamics }\end{array}\right.$.

\section{MEMBRANE PARADIGM}

In this section, we will begin with a brief discussion of the action formulation of the black hole membrane paradigm and then examine the electromagnetic membrane in the framework of NLED. The interested reader can find a detailed discussion of the action formulation of the membrane paradigm in [6].

The black hole has an event horizon, $\mathcal{H}$, which is a threedimensional null hypersurface with a null geodesic generator $l_{a}$. We then choose a timelike surface just outside $\mathcal{H}$, which is called the stretched horizon and denoted by $\mathcal{S}$. We regard $\mathcal{S}$ as the world tube of a family of fiducial observers, 
which have world lines $U^{a}$. The stretched horizon also possesses a spacelike outward-pointing normal vector $n_{a}$, which satisfies $n^{a} \nabla_{a} n^{c}=0$ on $\mathcal{S}$. Since the region inside the black hole cannot classically affect an outside observer $\mathcal{O}$, the classical equations of motion for $\mathcal{O}$ must be obtained by extremizing the action restricted to the spacetime outside the black hole, $S_{\text {out }}$. However, $S_{\text {out }}$ is not stationary on its own because there are no boundary conditions fixed at $\mathcal{H}$. To have the correct Euler-Lagrange equations, it is necessary to add a surface term $S_{\text {surf }}$ to $S_{\text {out }}$ to exactly cancel all the boundary terms. In practice, it is often more convenient to define $S_{\text {surf }}$ on $\mathcal{S}$. Consequently, the total action can be rewritten as

$$
S_{\text {tot }}=\left(S_{\text {out }}+S_{\text {surf }}\right)+\left(S_{\text {in }}-S_{\text {surf }}\right),
$$

where $\delta S_{\text {out }}+\delta S_{\text {surf }}=0$ will give the correct equations of motion outside $\mathcal{S}$. For the Maxwell action, the surface term can be interpreted as sources such as surface electric charges and currents [6].

For a nonlinear electromagnetic field $A_{a}$, the external action is given by Eq. (1):

$$
S_{\text {out }}=\int d^{4} x \sqrt{-g}\left[\mathcal{L}(s, p)+J^{a} A_{a}\right] .
$$

Integration by parts on the kinetic term of the nonlinear electromagnetic field leads to a variation at the boundary,

$$
-\int_{\mathcal{S}} d^{3} x \sqrt{-h} n_{b} G^{a b} \delta A_{a}
$$

where $h_{a b}=g_{a b}-n_{a} n_{b}$ is the induced metric on $\mathcal{S}$. To cancel this boundary contribution, we add a surface term $S_{\text {surf }}$,

$$
S_{\text {surf }}=\int_{\mathcal{S}} d^{3} x \sqrt{|h|} j_{\mathrm{s}}^{a} A_{a}
$$

where we define the membrane current as

$$
j_{\mathrm{s}}^{a}=G^{a b} n_{b} .
$$

The current $j_{\mathrm{s}}^{a}$ is on the stretched horizon since $n_{a} j_{\mathrm{s}}^{a}=0$. As in the Maxwell case, this surface term corresponds to the surface electric charge density $\rho=-j_{\mathrm{s}}^{a} U_{a}$ and current density $\mathbf{j}_{\mathrm{s}}^{a}=j_{\mathrm{s}}^{a}-\sigma U^{a}$. From Eq. (5), one can find a continuity equation for the membrane current $j_{\mathrm{s}}^{a}$ :

$$
\nabla_{a} j_{\mathrm{s}}^{a}=-J^{a} n_{a},
$$

where $-J^{a} n_{a}$ describes the charges crossing the stretched horizon.

We now consider a general black brane background, the metric of which takes the form

$$
\begin{aligned}
d s^{2} & =g_{a b} d x^{a} d x^{b}=g_{r r}(r) d r^{2}+g_{\mu \nu}(r) d x^{\mu} d x^{\nu} \\
& =-g_{t t}(r) d t^{2}+g_{r r}(r) d r^{2}+g_{A B}(r) d x^{A} d x^{B} \\
& =-g_{t t}(r) d t^{2}+g_{r r}(r) d r^{2}+g_{z z}(r)\left(d y^{2}+d z^{2}\right),
\end{aligned}
$$

where indices $\{a, b\}$ run over the $(3+1)$-dimensional bulk space; $\{\mu, \nu\}$ over a three-dimensional constant- $r$ hypersurface; and $\{A, B\}$ over spatial coordinates. We assume that there is an event horizon at $r=r_{h}$, where $g_{t t}(r)$ has a first order zero, $g_{r r}(r)$ has a first order pole, and $g_{z z}(r)$ is nonzero and finite. The Hawking temperature of the metric (25) is

$$
T=\frac{\sqrt{g_{t t}^{\prime}\left(r_{h}\right) g^{r r \prime}\left(r_{h}\right)}}{4 \pi} .
$$

We also assume that the couplings in $\mathcal{L}(s, p)$ only depend on $r$.

Now put the stretched horizon at $r=r_{0}$ with $r_{0}-r_{h} \ll r_{h}$. This stretched horizon would have

$$
n_{a}=\sqrt{g_{r r}\left(r_{0}\right)} \delta_{a r} \quad \text { and } \quad U_{a}=-\sqrt{g_{t t}\left(r_{0}\right)} \delta_{a t} .
$$

Thus, the membrane current (23) reduces to

$$
j_{\mathrm{s}}^{\mu}=\sqrt{g_{r r}\left(r_{0}\right)} G^{\mu r} .
$$

To find relations among $F_{a b}\left(r_{0}\right)$, we consider a radially freely falling observer in our background. It is easy to obtain the four-velocity vector of this infalling observer:

$$
U_{\mathrm{FFO}}^{a}=\left(\tilde{E} g_{t t}^{-1},-\tilde{E} g_{t t}^{-1} \sqrt{\frac{g_{t t}}{g_{r r}}} \sqrt{1-\tilde{E}^{-2} g_{t t}}, 0,0\right),
$$

where $\tilde{E}$ is the conserved energy per unit mass. The fact that $\tau$ is the proper time along the infalling world lines means that $U_{\mathrm{FFO}, a}$ is equal to the gradient of $\tau$,

$$
U_{\mathrm{FFO}, a}=-\partial_{\mu} \tau,
$$

from which one finds

$$
\frac{\partial \tau}{\partial t}=\tilde{E} \quad \text { and } \quad \frac{\partial \tau}{\partial r}=\tilde{E} \sqrt{\frac{g_{r r}}{g_{t t}}} \sqrt{1-\tilde{E}^{-2} g_{t t}} .
$$

Since this freely falling observer does not see the coordinate singularity at the horizon, the field strength observed by this observer must be regular. Relating $F^{\tau A}$ to $F^{r A}$ and $F^{t A}$, we obtain

$$
\begin{aligned}
F^{\tau A} & =\frac{\partial \tau}{\partial r} F^{r A}+\frac{\partial \tau}{\partial t} F^{t A} \\
& \Rightarrow \tilde{E}\left(\sqrt{g_{r r}} \sqrt{1-\tilde{E}^{-2} g_{t t}} F^{r A}+\sqrt{g_{t t}} F^{t A}\right)=F^{\tau A} \sqrt{g_{t t}},
\end{aligned}
$$


where $F^{\tau A}$ is finite at $r=r_{h}$. On the stretched horizon, $\sqrt{g_{t t}} F^{t A}$ is the electric field measured by the fiducial observers; hence, it would not go to zero as $r_{0} \rightarrow r_{h}$. Since $F^{\tau A} \sqrt{g_{t t}}$ goes to zero as $r_{0} \rightarrow r_{h}$, Eq. (32) leads to

$$
F^{r A}\left(r_{0}\right)=-\sqrt{\frac{g_{t t}\left(r_{0}\right)}{g_{r r}\left(r_{0}\right)}} F^{t A}\left(r_{0}\right),
$$

for $r_{0}-r_{h} \ll r_{h}$.

Using Eqs. (6), (28), and (33), we find that

$j_{\mathrm{s}}^{A}=\left.\mathcal{L}^{(1,0)}(s, p)\right|_{r=r_{0}} E^{A}-\left.[A B] \mathcal{L}^{(0,1)}(s, p)\right|_{r=r_{0}} E^{B}$,

where $\quad \mathcal{L}^{(1,0)}(s, p)=\partial \mathcal{L}(s, p) / \partial s \quad$ and $\quad \mathcal{L}^{(0,1)}(s, p)=$ $\partial \mathcal{L}(s, p) / \partial p$; the electric and magnetic fields measured by the fiducial observers on the stretched horizon are

$$
\begin{aligned}
& E^{a}=F^{t a}\left(r_{0}\right) \sqrt{g_{t t}\left(r_{0}\right)} \text { and }
\end{aligned}
$$

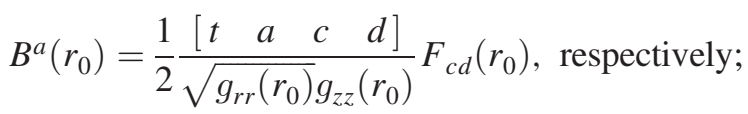

two variables $s$ and $p$ on the stretched horizon become

$s\left(r_{0}\right)=\frac{1}{2}\left(E^{r} E_{r}-B^{r} B_{r}\right) \quad$ and $\quad p\left(r_{0}\right)=-E^{r} B_{r}$.

Since $s$ and $p$ are scalars and the field strength observed by the freely falling observer is regular on the horizon, $s\left(r_{0}\right)$ and $p\left(r_{0}\right)$ stay finite as $r_{0} \rightarrow r_{h}$.

The conductivities of the stretched horizon can be read off from Eq. (34):

$$
\begin{aligned}
& \sigma_{\mathrm{s}}^{y y}=\sigma_{\mathrm{s}}^{z z}=\left.\mathcal{L}^{(1,0)}(s, p)\right|_{r=r_{0}} \quad \text { and } \\
& \sigma_{\mathrm{s}}^{z y}=-\sigma_{\mathrm{s}}^{y z}=\left.\mathcal{L}^{(0,1)}(s, p)\right|_{r=r_{0}},
\end{aligned}
$$

where $\sigma^{z y}$ is the surface Hall conductance. In MaxwellChern-Simons theory with $\mathcal{L}(s, p)=s+\theta p$, one has

$$
\sigma_{\mathrm{s}}^{y y}=\sigma_{\mathrm{s}}^{z z}=1 \quad \text { and } \quad \sigma_{\mathrm{s}}^{z y}=-\sigma_{\mathrm{s}}^{y z}=\theta,
$$

which agree with what was found in [12]. However, in NLED models, the conductivities of the stretched horizon usually depend on the external fields through $s\left(r_{0}\right)$ and $p\left(r_{0}\right)$. In particular, the conductivities only depend on the normal electric and magnetic fields measured by the fiducial observers on the stretched horizon. Note that the normal components of the electric and magnetic fields in an orthonormal frame of fiducial observers are the same as in those of freely falling observers. It is noteworthy that the surface charge density $\rho_{\mathrm{s}}$ of the stretched horizon can be related to $E_{r}$ and $B_{r}$ via

$$
\begin{aligned}
\rho_{\mathrm{s}} & =n_{a} D^{a} \\
& =\sqrt{g_{r r}\left(r_{0}\right)}\left[\left.\mathcal{L}^{(1,0)}(s, p)\right|_{r=r_{0}} E_{r}-\left.\mathcal{L}^{(0,1)}(s, p)\right|_{r=r_{0}} B_{r}\right] .
\end{aligned}
$$

Using this equation, we can rewrite the conductivities in terms of the surface charge density and normal magnetic field.

\section{INFALLING CHARGE IN RINDLER SPACE}

In this section, we will consider dropping a charged point particle into the horizon in Rindler space. Rindler space is a good approximation to the Schwarzschild geometry in the near-horizon region $r-2 M \ll 2 M$ and ignores the spatial curvature there. The metric of Rindler space takes the form

$$
d s^{2}=-r^{2} d \omega^{2}+d r^{2}+d y^{2}+d z^{2},
$$

which describes a portion of Minkowski space, namely, the Rindler wedge. Minkowski coordinates $t$ and $x$ can be defined by

$$
t=r \sinh \omega \quad \text { and } \quad x=r \cosh \omega
$$

to get the familiar Minkowski metric

$$
d s^{2}=-d t^{2}+d x^{2}+d y^{2}+d z^{2} .
$$

The Rindler coordinate has a coordinate singularity at $r=0$, where the determinant of the metric tensor becomes zero. In fact, there is an event horizon at $r=0$, which becomes $x=|t|$ in Minkowski coordinates and is the edge of the Rindler wedge. We will put the stretched horizon at $r=r_{0} \ll 1$, which has

$$
n^{a}=(0,1,0,0) \quad \text { and } \quad U^{a}=\left(r_{0}^{-1}, 0,0,0\right) .
$$

Without loss of generality, we can take a single charge to be static at position $x=a$ in Minkowski coordinates. In the Rindler coordinates, the charge is freely falling into the horizon. In this case, the magnetic and electric fields in Minkowski coordinates have been obtained in Sec. II and are given by Eqs. (16) and (17) with $r^{2}=(x-a)^{2}+y^{2}+z^{2}$, respectively. In Minkowski coordinates, the field strength is 


$$
\begin{aligned}
& F_{\mathrm{M}}^{t x}=-F_{\mathrm{M}}^{x t}=\frac{Q(x-a)}{4 \pi\left[(x-a)^{2}+y^{2}+z^{2}\right]^{\frac{3}{2}}} F\left(\frac{Q}{4 \pi\left[(x-a)^{2}+y^{2}+z^{2}\right]}\right), \\
& F_{\mathrm{M}}^{t y}=-F_{\mathrm{M}}^{y t}=\frac{Q y}{4 \pi\left[(x-a)^{2}+y^{2}+z^{2}\right]^{\frac{3}{2}}} F\left(\frac{Q}{4 \pi\left[(x-a)^{2}+y^{2}+z^{2}\right]}\right), \\
& F_{\mathrm{M}}^{t z}=-F_{\mathrm{M}}^{z t}=\frac{Q z}{4 \pi\left[(x-a)^{2}+y^{2}+z^{2}\right]^{\frac{3}{2}}} F\left(\frac{Q}{4 \pi\left[(x-a)^{2}+y^{2}+z^{2}\right]}\right),
\end{aligned}
$$

and all the other components are zero. Performing the change of coordinates to calculate $F_{\mathrm{R}}^{a b}$ leads to

$$
\begin{aligned}
j_{\mathrm{s}}^{\omega} & =\frac{Q\left(r_{0} \cosh \omega-a\right)}{4 \pi r_{0}\left[\left(r_{0} \cosh \omega-a\right)^{2}+y^{2}+z^{2}\right]^{\frac{3}{2}}}, \\
j_{\mathrm{s}}^{\rho} & =0, \\
j_{\mathrm{s}}^{y} & =\frac{Q}{4 \pi\left[\left(r_{0} \cosh \omega-a\right)^{2}+y^{2}+z^{2}\right]^{\frac{3}{2}}}\left[y \sinh \omega-\left.z \cosh \omega \frac{\mathcal{L}^{(0,1)}(s, 0)}{\mathcal{L}^{(1,0)}(s, 0)}\right|_{r=r_{0}}\right], \\
j_{\mathrm{s}}^{z} & =\frac{Q}{4 \pi\left[\left(r_{0} \cosh \omega-a\right)^{2}+y^{2}+z^{2}\right]^{\frac{3}{2}}}\left[z \sinh \omega+\left.y \cosh \omega \frac{\mathcal{L}^{(0,1)}(s, 0)}{\mathcal{L}^{(1,0)}(s, 0)}\right|_{r=r_{0}}\right]
\end{aligned}
$$

where we use $F(X) \mathcal{L}^{(1,0)}\left(\frac{X^{2}}{2} F^{2}(X), 0\right)=1$ to eliminate the function $F(X)$, and

$$
s\left(r_{0}\right)=\frac{Q^{2}}{32 \pi^{2}\left[\left(r_{0} \cosh \omega-a\right)^{2}+y^{2}+z^{2}\right]^{2}} F^{2}\left(\frac{Q}{4 \pi\left[\left(r_{0} \cosh \omega-a\right)^{2}+y^{2}+z^{2}\right]}\right) .
$$

For Maxwell-Chern-Simons theory, we also reproduce the results in [12]. A fiducial observer will measure a surface charge density $\rho_{\mathrm{s}}=-j_{\mathrm{s}}^{a} U_{a}=r_{0} j_{\mathrm{s}}^{\omega}$. In NLED, the surface charge density $\rho_{\mathrm{S}}$ of the stretched horizon is exactly the same as in Maxwell theory. In NLED, there are no corrections to $\rho_{\mathrm{s}}$, but the surface currents $j_{\mathrm{s}}^{y}$ and $j_{\mathrm{s}}^{z}$ could receive corrections.

Let us study the scrambling of point charges on the stretched horizon for large Rindler time. When $\omega \gg 1$, we obtain

$$
\begin{aligned}
& \rho_{\mathrm{s}}=\frac{r_{0} Q e^{-2 \omega}}{\pi\left(r_{0}^{2}+r_{\perp}^{2}\right)^{\frac{3}{2}}}, \\
& j_{\mathrm{s}}^{y}=\frac{Q}{\pi\left(r_{0}^{2}+r_{\perp}^{2}\right)^{\frac{3}{2}}}\left[y-\frac{\theta\left(r_{0}\right)}{g\left(r_{0}\right)} z\right] \\
& j_{\mathrm{s}}^{z}=\frac{Q}{\pi\left(r_{0}^{2}+r_{\perp}^{2}\right)^{\frac{3}{2}}}\left[z+\frac{\theta\left(r_{0}\right)}{g\left(r_{0}\right)} y\right],
\end{aligned}
$$

where $r_{\perp}^{2}=4 e^{-2 \omega}\left(y^{2}+z^{2}\right)$, and we use $s\left(r_{0}\right) \sim e^{-2 \omega}$ in this limit. Whenever $\left.\theta\left(r_{0}\right) \equiv \mathcal{L}^{(0,1)}(0,0)\right|_{r=r_{0}} \neq 0$, effects of NLED would change the way the charge scrambles but not the scrambling time. In this case, there is the presence of vortices on the stretched horizon [12].

\section{DC CONDUCTIVITY FROM GAUGE/GRAVITY DUALITY}

Under the long-wavelength and low-frequency limit, it is expected that there are connections between the near-horizon region geometry of the bulk gravity and the dual field theory living on the boundary. Observing that the currents in the boundary theory could be identified with radially independent quantities in the bulk, the authors of [17] found that the low-frequency limit of linear response of the boundary theory could be determined by the membrane paradigm fluid on the stretched horizon. In particular, they derived an expression for the DC conductivity of the boundary theory in terms of geometric quantities evaluated at the horizon. In [17], the conserved current in the boundary theory was dual to a Maxwell field in the bulk. In this section, we will follow the method in [17] to calculate the DC conductivities of the conserved current in the boundary theory, which is dual to a NLED field in bulk.

We now consider a probe NLED field in the background of a $(3+1)$-dimensional black brane with the metric $(25)$. For simplicity, we assume that this black brane is electrically neutral with trivial background configuration of the NLED field. This black brane describes an equilibrium state at finite temperature $T$, which is given by Eq. (26). The action of the NLED field is

$$
S=\int d^{4} x \sqrt{-g} \mathcal{L}(s, p)
$$

This NLED field is a U(1) gauge field and dual to a conserved current $\mathcal{J}^{\mu}$ in the boundary theory. The corresponding $\mathrm{AC}$ conductivities are given by 


$$
\left\langle\mathcal{J}^{A}\left(k_{\mu}\right)\right\rangle=\sigma^{A B}\left(k_{\mu}\right) F_{B t}\left(r_{B}\right),
$$

where the boundary theory lives at $r=r_{B} \rightarrow \infty$. The DC conductivity is obtained in the long-wavelength and lowfrequency limit:

$$
\sigma^{A B}=\lim _{\omega \rightarrow 0} \lim _{\vec{k} \rightarrow 0} \sigma^{A B}\left(k_{\mu}\right) .
$$

Apart from varying the action, we can also derive the equations of motion using Hamiltonian formulation. Using gauge choice $A_{r}=0$, we will find the equations of motion for $A_{\mu}$ in a Hamiltonian form. From the action (46), the conjugate momentum of the field $A_{\mu}$ with respect to $r$ foliation is given by

$$
\Pi^{\mu}=\frac{\delta S}{\delta\left(\partial_{r} A_{\mu}\right)}=-\sqrt{-g} G^{r \mu}
$$

where $G^{r \mu}$ are defined in Eq. (6). Since $G^{r \mu}$ are functions of $F^{r \mu}$ and $F^{\mu \nu}$, one could solve Eq. (49) to find an expression for $F^{r \mu}$ in terms of $\Pi^{\mu}$ and $F^{\mu \nu}$ :

$$
F^{r \mu}=F^{r \mu}\left(\Pi^{\nu}, F^{\rho \sigma}\right),
$$

where, as will be shown later, the exact form of the function $F^{r \mu}\left(\Pi^{\nu}, F^{\rho \sigma}\right)$ is not important for our discussion. So the Hamiltonian is given by

$$
\begin{aligned}
H & =\int d^{4} x \sqrt{-g} \mathcal{H}\left(\Pi^{\nu}, F^{\rho \sigma}\right) \\
& =\int d^{4} x \sqrt{-g}\left[F_{r \mu}\left(\Pi^{\nu}, F^{\rho \sigma}\right) \Pi^{\mu}-\mathcal{L}(s, p)\right],
\end{aligned}
$$

where we use Eq. (50) to rewrite $s$ and $p$ in terms of $\Pi^{\nu}$ and $F^{\rho \sigma}$. Varying the Hamiltonian with respect to $A_{\mu}$, we write the equations of motion for $A_{\mu}$ in a Hamiltonian form as

$$
\partial_{r} \Pi^{\mu}=-2 \sqrt{-g} \partial_{\nu}\left[\frac{\partial \mathcal{H}\left(\Pi^{\eta}, F^{\rho \sigma}\right)}{\partial F_{\nu \mu}}\right] .
$$

Moreover, the Bianchi identity gives

$$
\partial_{r} F_{\mu \nu}+\partial_{\mu} F_{\nu r}\left(\Pi^{\eta}, F^{\rho \sigma}\right)+\partial_{\nu} F_{r \mu}\left(\Pi^{\eta}, F^{\rho \sigma}\right)=0 .
$$

In the long-wavelength and low-frequency limit, i.e., $\omega \rightarrow$ 0 and $\vec{k} \rightarrow 0$ with $F^{\rho \sigma}$ and $\Pi^{\eta}$ fixed, Eqs. (52) and (53) become

$$
\partial_{r} \Pi^{\mu}=0 \quad \text { and } \quad \partial_{r} F_{\mu \nu}=0 .
$$

Now we discuss boundary conditions for $F_{a b}$ on the stretched horizon at $r=r_{0} \rightarrow r_{h}$ and the boundary of bulk at $r=r_{B} \rightarrow \infty$. On the stretched horizon, $s$ and $p$ become

$$
\begin{aligned}
& s\left(r_{0}\right)=\frac{1}{2}\left[g_{r r}\left(r_{0}\right) g_{t t}\left(r_{0}\right) F^{r t}\left(r_{0}\right)^{2}-\frac{F_{y z}^{2}}{g_{z z}^{2}\left(r_{0}\right)}\right], \\
& p\left(r_{0}\right)=\frac{g_{r r}\left(r_{0}\right) g_{t t}\left(r_{0}\right)}{\sqrt{-g\left(r_{0}\right)}} F^{r t}\left(r_{0}\right) F_{y z},
\end{aligned}
$$

where we use Eq. (33) to express $F^{r A}\left(r_{0}\right)$ in terms of $F^{t A}\left(r_{0}\right)$, and $F_{y z}$ is an $r$-independent quantity. Using Eqs. (28) and (49), one can relate $\Pi^{A}\left(r_{0}\right)$ to $j_{\mathrm{s}}^{A}$ :

$$
\begin{aligned}
\Pi^{A}\left(r_{0}\right) & =\sqrt{g_{t t}\left(r_{0}\right)} g_{z z}\left(r_{0}\right) j_{s}^{A} \\
& =\left.\mathcal{L}^{(1,0)}(s, p)\right|_{r=r_{0}} F_{A t}-\left.[A B] \mathcal{L}^{(0,1)}(s, p)\right|_{r=r_{0}} F_{B t}
\end{aligned}
$$

where $F_{A t}$ is also $r$-independent. On the boundary of the bulk, it was shown in $[17,44]$ that a one-point function of $\mathcal{J}^{A}$ in the presence of source $F_{\mu v}$ can be written as

$$
\left\langle\mathcal{J}^{\mu}\right\rangle=\Pi^{\mu}\left(r_{B}\right)
$$

Since $\Pi^{A}$ and $F_{A t}$ are $r$-independent in the zero-momentum limit, we can use Eqs. (56) and (57) to show that

$$
\begin{aligned}
\left\langle\mathcal{J}^{A}\left(k_{\mu} \rightarrow 0\right)\right\rangle= & \left.\mathcal{L}^{(1,0)}(s, p)\right|_{r=r_{0}} F_{A t}\left(k_{\mu} \rightarrow 0\right) \\
& -\left.[A B] \mathcal{L}^{(0,1)}(s, p)\right|_{r=r_{0}} F_{B t}\left(k_{\mu} \rightarrow 0\right) .
\end{aligned}
$$

Comparing Eq. (47) with Eq. (58), we can read off the DC conductivities in the dual theory:

$$
\begin{aligned}
& \sigma^{y y}=\sigma^{z z}=\left.\mathcal{L}^{(1,0)}(s, p)\right|_{r=r_{h}} \text { and } \\
& \sigma^{z y}=-\sigma^{y z}=\left.\mathcal{L}^{(0,1)}(s, p)\right|_{r=r_{h}},
\end{aligned}
$$

where we take the limit $r \rightarrow r_{h}$. Note that the DC conductivity in NLED just with $s$ was also obtained in [39], where their Eq. (30) in the probe case agrees with our expression for $\sigma^{y y}$ in Eqs. (59).

To express $F^{r t}\left(r_{h}\right)$ in terms of quantities in the boundary theory, we can use the following formula,

$$
\Pi^{t}\left(r_{h}\right)=\Pi^{t}(r \rightarrow \infty)=\left\langle\mathcal{J}^{0}\right\rangle=\rho,
$$

where $\rho$ can be interpreted as the charge density in the dual field theory. Equation (60) becomes

$$
\begin{gathered}
\left.\sqrt{\eta} g_{z z}\left(r_{h}\right) \mathcal{L}^{(1,0)}(s, p)\right|_{r=r_{h}} F^{r t}\left(r_{h}\right) \\
+\left.\mathcal{L}^{(0,1)}(s, p)\right|_{r=r_{h}} F_{y z}=-\rho,
\end{gathered}
$$

where Eqs. (55) give 




(a) Plot of $\sigma^{y y}$ against $\rho$ and $F_{y z}$ for Born-Infeld electrodynamics

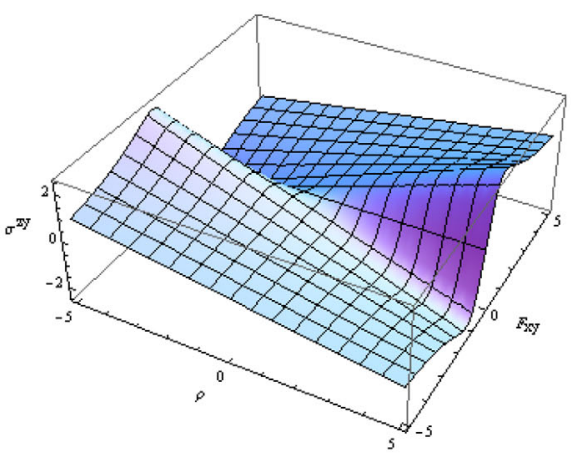

(b) Plot of $\sigma^{y z}$ against $\rho$ and $F_{y z}$ for Born-Infeld electrodynamics

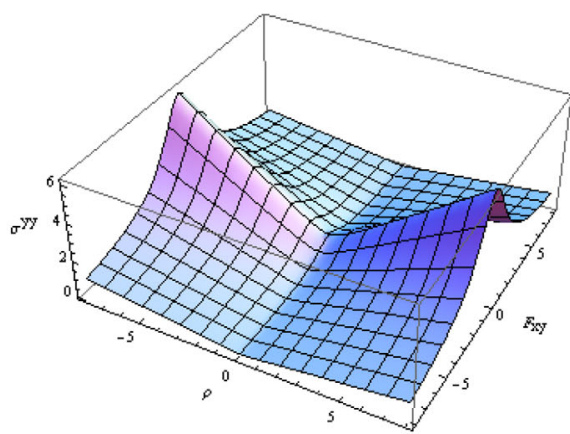

(c) Plot of $\sigma^{y y}$ against $\rho$ and $F_{y z}$ for Logarithmic electrodynamics

FIG. 1. Plots of the DC conductivities of the conserved current dual to the bulk electromagnetic field in Born-Infeld and Logarithmic electrodynamics. Here we set $b^{2} g_{z z}^{2}\left(r_{h}\right)=1$.

$$
\begin{aligned}
& s\left(r_{h}\right)=\frac{1}{2}\left[\eta F^{r t}\left(r_{h}\right)^{2}-\frac{F_{y z}^{2}}{g_{z z}^{2}\left(r_{h}\right)}\right], \\
& p\left(r_{h}\right)=\frac{\sqrt{\eta}}{g_{z z}\left(r_{h}\right)} F^{r t}\left(r_{h}\right) F_{y z} .
\end{aligned}
$$

One could solve Eq. (61) to express $F^{r t}\left(r_{h}\right)$ in terms of $\rho$ and $F_{y z}$ and plug this expression into Eq. (59) to write $\sigma^{A B}$ in terms of $\rho$ and $F_{y z}$. Note that $F_{y z}$ can be treated as the magnetic field in the $(2+1)$-dimensional boundary theory, in which the magnetic field is a scalar field.

Let us consider $\sigma^{A B}$ in the Born-Infeld and logarithmic electrodynamics discussed above. We find that, for BornInfeld electrodynamics,

$$
\begin{aligned}
& \sigma^{y y}=\sigma^{z z}=\frac{\sqrt{1+F_{y z}^{2} /\left[b^{2} g_{z z}^{2}\left(r_{h}\right)\right]+\rho^{2} /\left[b^{2} g_{z z}^{2}\left(r_{h}\right)\right]}}{1+F_{y z}^{2} /\left[b^{2} g_{z z}^{2}\left(r_{h}\right)\right]}, \\
& \sigma^{y z}=-\sigma^{z y}=\frac{\rho F_{y z} /\left[b^{2} g_{z z}^{2}\left(r_{h}\right)\right]}{1+F_{y z}^{2} /\left[b^{2} g_{z z}^{2}\left(r_{h}\right)\right]},
\end{aligned}
$$

and for logarithmic electrodynamics

$$
\begin{aligned}
& \sigma^{y y}=\sigma^{z z}=\frac{1+\sqrt{1+\left\{2+F_{y z}^{2} /\left[b^{2} g_{z z}^{2}\left(r_{h}\right)\right]\right\} \rho^{2} /\left[g_{z z}^{2}\left(r_{h}\right) b^{2}\right]}}{2+F_{y z}^{2} /\left[b^{2} g_{z z}^{2}\left(r_{h}\right)\right]}, \\
& \sigma^{z y}=-\sigma^{y z}=0 .
\end{aligned}
$$

Note that the DC conductivity matrix of a holographic Dirac-Born-Infeld model in the probe limit has been calculated in [42]. Equations (3.1) and (3.2) with $S=0$ in [42] turn out to be the same as our results in (63). In Fig. 1, we plot the DC conductivities versus $\rho$ and $F_{y z}$, of the conserved current dual to the bulk electromagnetic field in both Born-Infeld and logarithmic electrodynamics. The parameter $b^{2} g_{z z}^{2}\left(r_{h}\right)$ sets a scale in the dual field theory. When $\rho^{2}, F_{y z}^{2} \ll b^{2} g_{z z}^{2}\left(r_{h}\right)$, we practically reproduce the results for Maxwell theory. On the other hand, effects of nonlinearity of the electromagnetic fields start to play an important role when $\rho^{2}$ or $F_{y z}^{2}$ is around the scale $b^{2} g_{z z}^{2}\left(r_{h}\right)$. At zero charge density, the diagonal components of the DC conductivities in both Born-Infeld and logarithmic electrodynamics are nonzero. These nonzero values can be interpreted as incoherent contributions [45], known as the charge conjugation symmetric terms, which are independent of the charge density $\rho$. As shown in Fig. 1, the diagonal DC conductivity $\sigma^{y y}$ increases with increasing $|\rho|$ at constant $F_{y z}$, which is a feature similar to the Drude metal. For the Drude metal, a larger charge density provides more available mobile charge carriers to efficiently transport charge. At constant $\rho, \sigma^{y y}$ decreases with increasing $\left|F_{y z}\right|$, which means a positive magnetoresistance.

Since $r_{h}$ is related to the Hawking temperature $T$ by Eq. (26), we can now discuss the temperature dependence of the conductivities. For simplicity and concreteness, we consider the Schwarzschild AdS black brane

$d s^{2}=-\left(r^{2}-r_{h}^{3} / r\right) d t^{2}+\frac{d r^{2}}{\left(r^{2}-r_{h}^{3} / r\right)}+r^{2}\left(d y^{2}+d z^{2}\right)$,

where we take the AdS radius $L=1$, and $r_{h}$ determines the Hawking temperature of the black brane:

$$
T=\frac{3 r_{h}}{4 \pi} .
$$

Therefore, we obtain that, for Born-Infeld electrodynamics,

$$
\begin{gathered}
\sigma^{y y}=\sigma^{z z}=\frac{\sqrt{1+\lambda F_{y z}^{2} / T^{4}+\lambda \rho^{2} / T^{4}}}{\lambda F_{y z}^{2} / T^{4}+1} \\
\sigma^{y z}=-\sigma^{z y}=\frac{\lambda \rho F_{y z} / T^{4}}{1+\lambda F_{y z}^{2} / T^{4}},
\end{gathered}
$$




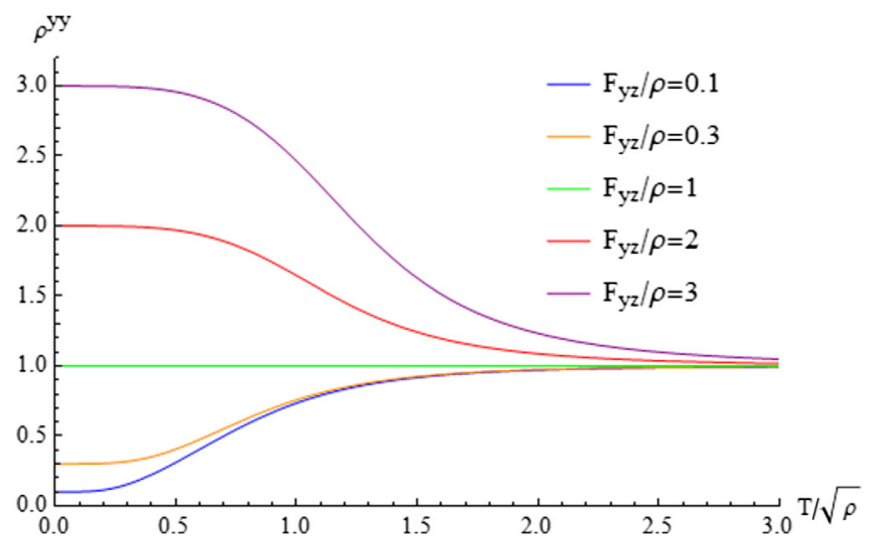

FIG. 2. Plot of $\rho^{y y}$ versus $T / \sqrt{\rho}$ in logarithmic electrodynamics.

and for logarithmic electrodynamics,

$$
\sigma^{y y}=\sigma^{z z}=\frac{1+\sqrt{1+\left(2+\lambda F_{y z}^{2} / T^{4}\right) \lambda \rho^{2} / T^{4}}}{2+\lambda F_{y z}^{2} / T^{4}},
$$

where $\lambda \equiv b^{-2}(4 \pi / 3)^{-4}$ is a parameter associated with the conserved current $\mathcal{J}^{\mu}$ in the boundary theory. In the high temperature limit, we would recover the results for Maxwell theory. When $T^{4} \ll \rho^{2} / b^{2}$ and $F_{y z}^{2} / b^{2}$, the low temperature behavior of $\sigma^{A B}$ is

$\sigma^{y y}=\sigma^{z z} \sim T^{2} \quad$ and

$\sigma^{z y}=-\sigma^{y z} \sim T^{0}$ for Born-Infeld electrodynamics,

and

$$
\sigma^{y y}=\sigma^{z z} \sim T^{0} \text { for logarithmic electrodynamics. }
$$

One can define a metal and an insulator for $d \rho^{y y} / d T>0$ and $d \rho^{y y} / d T<0$, respectively, where the resistivity matrix $\left\{\rho^{A B}\right\}$ is the inverse of the conductivity matrix $\left\{\sigma^{A B}\right\}$. The metal-insulator transition in Born-Infeld electrodynamics has been discussed in [43]. So we here focus on logarithmic electrodynamics. In Fig. 2, we plot $\rho^{y y}$ versus $T / \sqrt{\rho}$ for various values of $F_{y z} / \sqrt{\rho}$. The temperature dependence of $\rho^{y y}$ is similar to the case with a larger value of the momentum dissipation parameter in [43]. One has an insulator phase for $F_{y z}<\rho$ and a metal one for $F_{y z}>\rho$. A metal-insulator transition could occur at $F_{y z}=\rho$, where $\rho^{y y}=1$ is independent of the temperature.

\section{DISCUSSION AND CONCLUSION}

In the first part of this paper, we have used the membrane paradigm to study the electromagnetic membrane of black holes in NLED. In the membrane paradigm, the stretched horizon is regarded as a boundary hypersurface with the surface charge and current, which terminate the normal $D$ and tangential $H$ fields in the region exterior to the horizon, and annul them in the interior region. For Maxwell theory, it is well known that the horizon can be interpreted as an ohmic conductor with a constant resistivity. It was shown in [12] that the horizon behaved as a Hall conductor with surface Hall conductance in Maxwell-Chern-Simons theory. We derived the conductivities of the surface current for a general NLED and found that the conductivities usually depended on the normal electric and magnetic fields on the stretched horizon. We also showed that there was Hall conductance for the stretched horizon when $\mathcal{L}^{(0,1)}(s, p)$ was not zero on the horizon.

To study the effects of NLED on charges scrambling on the stretched horizon, we considered a simple scenario, in which a charged point particle freely falls into the horizon in Rindler space. Our results showed that, during the free falling, the surface charge density in NLED was the same as in Maxwell theory. However, the effects of NLED play a role in the surface current density. In particular, when $\mathcal{L}^{(0,1)}(s, p)$ did not vanish on the horizon, there would be the presence of vortices. In the late time limit, NLED would not change the scrambling time. This is expected since the electric field becomes smaller and smaller in this limit, and we assume that NLED would reduce to Maxwell-ChernSimons theory for small fields. In [12], it was found that the $\theta$-term only changed the way the charge scrambled but not the scrambling time in Maxwell-Chern-Simons theory. If some NLED differs from Maxwell-Chern-Simons theory in the IR limit, one would expect the scrambling time to be changed in this NLED.

In the second part of this paper, we used the membrane paradigm approach of [17] to calculate DC conductivities of a conserved current in a field theory living on the boundary of some black brane. We assumed that this conserved current was dual to a probe NLED field in the bulk. We found that the conjugate momentum of the NLED field encoded the information about the conductivities both on the stretched horizon and in the boundary theory and, in the zero frequency limit, did not evolve in the radial direction. Therefore, we showed that these DC conductivities depended only on the geometry and NLED fields at the black hole horizon, not on these of the whole bulk geometry. Relating electromagnetic quantities at the horizon to these in the boundary theory, we also showed that the DC conductivities usually depended on the probe charge density and magnetic field in the boundary theory.

We conclude this paper with a few remarks. First, we showed that the DC conductivities depended on the values of the couplings in NLED at the horizon. However, the authors of [17] showed that, in Maxwell-Chern-Simons theory, the Hall conductivity $\sigma^{y z}$ was determined by the value of $\theta$ coupling at the boundary of the bulk. We think that the discrepancy comes from the possibility that the authors of [17] failed to realize that the first term on the 
left-hand side of Eq. (53) in [17] was no longer $r$ independent in Maxwell-Chern-Simons theory. On the stretched horizon, this term only contributes to the diagonal components of the conductivities. However, this term is now $r$-dependent and would contribute to the off-diagonal components as well as the diagonal ones on the boundary of the bulk. In other words, the Hall conductivity of the boundary theory receives contributions from both terms on the left-hand side of Eq. (52), not just the first one. These two contributions indeed make the Hall conductivity depend on the value of $\theta$ coupling at the horizon. This incorrect statement of [17] has also been noted in [25], where the authors found that the $\theta$ parameter could vanish on the boundary with nonvanishing values on the horizon, hence giving rise to nonvanishing Hall conductivity.

Second, one usually only turns on the electric field in the boundary theory to calculate the holographic conductivities due to difficulties of solving the differential equations. On the other hand, the membrane paradigm provides a simple way to obtain the dependence of holographic DC conductivities on the electromagnetic quantities in the boundary theory, e.g., the charge density and magnetic field. Our analysis was carried out in the long-wavelength and low-frequency limit, which corresponds to an equilibrium and homogeneous state. In particular, the charge density and magnetic field in the boundary theory are kept fixed, time independent, and homogeneous in this limit.

Finally, we only considered a neutral black brane, which is dual to a boundary theory without a background charge density. As shown in [19], the low-frequency behavior of the conductivities depends crucially on whether there is a background charge density. It is very interesting to study the behavior of DC conductivities in a boundary theory with a nonvanishing background charge density, which is dual to a NLED charged black hole.

\section{ACKNOWLEDGMENTS}

We are grateful to Houwen $\mathrm{Wu}$, Zheng Sun, Jerome Gauntlett, Aristomenis Donos, Li Li, and Matteo Baggioli for useful discussions and valuable comments. This work is supported in part by NSFC (Grants No. 11005016, No. 11175039, and No. 11375121).
[1] K. S. Thorne and D. MacDonald, Electrodynamics in curved spacetime: $3+1$ formulation, Mon. Not. R. Astron. Soc. 198, 339 (1982).

[2] D. MacDonald and K. S. Thorne, Black-hole electrodynamics: An absolute-space/universal-time formulation, Mon. Not. R. Astron. Soc. 198, 345 (1982).

[3] D. MacDonald and W. M. Suen, Membrane viewpoint on black holes: Dynamical electromagnetic fields near the horizon, Phys. Rev. D 32, 828 (1984).

[4] R. H. Price and K. S. Thorne, Membrane viewpoint on black holes: Properties and evolution of the stretched horizon, Phys. Rev. D 33, 915 (1986).

[5] W. M. Suen, R. H. Price, and I. H. Redmount, Membrane viewpoint on black holes: Gravitational perturbations of the horizon, Phys. Rev. D 37, 2761 (1988).

[6] M. Parikh and F. Wilczek, An action for black hole membranes, Phys. Rev. D 58, 064011 (1998).

[7] J. Masso, E. Seidel, W. M. Suen, and P. Walker, Event horizons in numerical relativity 2.: Analyzing the horizon, Phys. Rev. D 59, 064015 (1999).

[8] S. S. Komissarov, Electrodynamics of black hole magnetospheres, Mon. Not. R. Astron. Soc. 350, 407 (2004).

[9] R. F. Penna, R. Narayan, and A. Sadowski, General relativistic magnetohydrodynamic simulations of BlandfordZnajek jets and the membrane paradigm, Mon. Not. R. Astron. Soc. 436, 3741 (2013).

[10] L. Susskind and J. S. Lindesay, Black Holes, Information, and the String Theory Revolution: The Holographic Universe (Singapore, World Scientific, 2005).
[11] Y. Sekino and L. Susskind, Fast scramblers, J. High Energy Phys. 10 (2008) 065.

[12] W. Fischler and S. Kundu, Hall scrambling on black hole horizons, Phys. Rev. D 92, 046008 (2015).

[13] T. Banks, W. Fischler, S. H. Shenker, and L. Susskind, M theory as a matrix model: A conjecture, Phys. Rev. D 55, 5112 (1997).

[14] J. M. Maldacena, The large N limit of superconformal field theories and supergravity, Int. J. Theor. Phys. 38, 1113 (1999); Adv. Theor. Math. Phys. 2, 231 (1998).

[15] P. Kovtun, D. T. Son, and A. O. Starinets, Holography and hydrodynamics: Diffusion on stretched horizons, J. High Energy Phys. 10 (2003) 064.

[16] P. Kovtun, D. T. Son, and A. O. Starinets, Viscosity in Strongly Interacting Quantum Field Theories from Black Hole Physics, Phys. Rev. Lett. 94, 111601 (2005).

[17] N. Iqbal and H. Liu, Universality of the hydrodynamic limit in AdS/CFT and the membrane paradigm, Phys. Rev. D 79, 025023 (2009).

[18] I. Bredberg, C. Keeler, V. Lysov, and A. Strominger, Wilsonian approach to fluid/gravity duality, J. High Energy Phys. 03 (2011) 141.

[19] M. Blake and D. Tong, Universal resistivity from holographic massive gravity, Phys. Rev. D 88, 106004 (2013).

[20] A. Donos and J. P. Gauntlett, Thermoelectric DC conductivities from black hole horizons, J. High Energy Phys. 11 (2014) 081.

[21] S. Cremonini, H. S. Liu, H. Lu, and C. N. Pope, DC conductivities from non-relativistic scaling geometries with 
momentum dissipation, J. High Energy Phys. 04 (2017) 009.

[22] N. Bhatnagar and S. Siwach, DC conductivity with external magnetic field in hyperscaling violating geometry, Int. J. Mod. Phys. A33, 1850028 (2018).

[23] A. Donos and J. P. Gauntlett, Navier-Stokes equations on black hole horizons and DC thermoelectric conductivity, Phys. Rev. D 92, 121901 (2015).

[24] E. Banks, A. Donos, and J. P. Gauntlett, Thermoelectric DC conductivities and Stokes flows on black hole horizons, J. High Energy Phys. 10 (2015) 103.

[25] A. Donos, J. P. Gauntlett, T. Griffin, N. Lohitsiri, and L. Melgar, Holographic DC conductivity and Onsager relations, J. High Energy Phys. 07 (2017) 006.

[26] W. Heisenberg and H. Euler, Consequences of Dirac's theory of positrons, Z. Phys. 98, 714 (1936).

[27] M. Born and L. Infeld, Foundations of the new field theory, Proc. R. Soc. A 144, 425 (1934).

[28] H. H. Soleng, Charged black points in general relativity coupled to the logarithmic U(1) gauge theory, Phys. Rev. D 52, 6178 (1995).

[29] E. Ayon-Beato and A. Garcia, Regular Black Hole in General Relativity Coupled to Nonlinear Electrodynamics, Phys. Rev. Lett. 80, 5056 (1998).

[30] R. G. Cai, D. W. Pang, and A. Wang, Born-Infeld black holes in (A)dS spaces, Phys. Rev. D 70, 124034 (2004).

[31] H. Maeda, M. Hassaine, and C. Martinez, Lovelock black holes with a nonlinear Maxwell field, Phys. Rev. D 79, 044012 (2009).

[32] S. H. Hendi, B. Eslam Panah, S. Panahiyan, and A. Sheykhi, Dilatonic BTZ black holes with power-law field, Phys. Lett. B 767, 214 (2017).
[33] R. G. Cai and Y. W. Sun, Shear viscosity from AdS BornInfeld black holes, J. High Energy Phys. 09 (2008) 115.

[34] J. Jing and S. Chen, Holographic superconductors in the Born-Infeld electrodynamics, Phys. Lett. B 686, 68 (2010).

[35] J. Jing, Q. Pan, and S. Chen, Holographic superconductors with Power-Maxwell field, J. High Energy Phys. 11 (2011) 045.

[36] S. Gangopadhyay and D. Roychowdhury, Analytic study of Gauss-Bonnet holographic superconductors in Born-Infeld electrodynamics, J. High Energy Phys. 05 (2012) 156.

[37] D. Roychowdhury, Effect of external magnetic field on holographic superconductors in presence of nonlinear corrections, Phys. Rev. D 86, 106009 (2012).

[38] A. Dehyadegari, M. Kord Zangeneh, and A. Sheykhi, Holographic conductivity in the massive gravity with power-law Maxwell field, Phys. Lett. B 773, 344 (2017).

[39] M. Baggioli and O. Pujolas, On effective holographic Mott insulators, J. High Energy Phys. 12 (2016) 107.

[40] A. Karch and A. O'Bannon, Metallic AdS/CFT, J. High Energy Phys. 09 (2007) 024.

[41] A. O'Bannon, Hall conductivity of flavor fields from AdS/CFT, Phys. Rev. D 76, 086007 (2007).

[42] E. Kiritsis and L. Li, Quantum criticality and DBI magnetoresistance, J. Phys. A 50, 115402 (2017).

[43] S. Cremonini, A. Hoover, and L. Li, Backreacted DBI magnetotransport with momentum dissipation, J. High Energy Phys. 10 (2017) 133.

[44] S. A. Hartnoll, Lectures on holographic methods for condensed matter physics, Classical Quantum Gravity 26, 224002 (2009).

[45] R. A. Davison and B. Goutéraux, Dissecting holographic conductivities, J. High Energy Phys. 09 (2015) 090. 